\title{
Status and perspectives of the BAIKAL-GVD project
}

\author{
A.D. Avrorin ${ }^{1}$, A.V. Avrorin ${ }^{1}$, V.M. Aynutdinov ${ }^{1}$, R. Bannash ${ }^{7}$, I.A. Belolaptikov², \\ D.Yu. Bogorodsky ${ }^{3}$, V.B. Brudanin ${ }^{2}$, N.M. Budnev ${ }^{3}$, I.A. Danilchenko ${ }^{1}$, G.V. Domogatsky ${ }^{1}$, \\ A.A. Doroshenko ${ }^{1}$, A.N. Dyachok ${ }^{3}$, Zh.-A.M. Dzhilkibaev ${ }^{1, a}$, S.V. Fialkovsky ${ }^{5}$, \\ A.R. Gafarov ${ }^{3}$, O.N. Gaponenko ${ }^{1}$, K.V. Golubkov ${ }^{1}$, T.I. Gress ${ }^{3}$, Z. Honz ${ }^{2}$, K.G. Kebkal ${ }^{7}$, \\ O.G. Kebkal ${ }^{7}$, K.V. Konischev ${ }^{2}$, E.N. Konstantinov ${ }^{3}$, A.V. Korobchenko ${ }^{3}$, A.P. Koshechkin ${ }^{1}$, \\ F.K. Koshel ${ }^{1}$, A.V. Kozhin ${ }^{4}$, V.F. Kulepov ${ }^{5}$, D.A. Kuleshov ${ }^{1}$, V.I. Ljashuk ${ }^{1}$, M.B. Milenin ${ }^{5}$, \\ R.A. Mirgazov ${ }^{3}$, E.R. Osipova ${ }^{4}$, A.I. Panfilov ${ }^{1}$, L.V. Pan'kov' ${ }^{3}$, A.A. Perevalov ${ }^{3}$, \\ E.N. Pliskovsky², M.I. Rozanov ${ }^{6}$, V.Yu. Rubtzov ${ }^{3}$, E.V. Rjabov ${ }^{3}$, B.A. Shaybonov' ${ }^{2}$, \\ A.A. Sheifler ${ }^{1}$, A.V. Skurihin ${ }^{4}$, A.A. Smagina ${ }^{2}$, O.V. Suvorova ${ }^{1}$, V.A. Tabolenko, \\ B.A. Tarashansky ${ }^{3}$, S.A. Yakovlev ${ }^{7}$, A.V. Zagorodnikov ${ }^{3}$, V.A. Zhukov ${ }^{1}$, and V.L. Zurbanov ${ }^{3}$ \\ ${ }^{1}$ Institute for Nuclear Research, 60th October Anniversary pr. 7A, Moscow 117312, Russia \\ 2 Joint Institute for Nuclear Research, Dubna 141980, Russia \\ ${ }^{3}$ Irkutsk State University, Irkutsk 664003, Russia \\ ${ }^{4}$ Skobeltsyn Institute of Nuclear Physics MSU, Moscow 119991, Russia \\ ${ }^{5}$ Nizhni Novgorod State Technical University, Nizhni Novgorod 603950, Russia \\ ${ }^{6}$ St. Petersburg State Marine University, St. Petersburg 190008, Russia \\ ${ }^{7}$ EvoLogics GmbH, Berlin, Germany
}

\begin{abstract}
The neutrino telescope Baikal-GVD in Lake Baikal will be a research infrastructure aimed mainly at studying astrophysical neutrino fluxes. The telescope will consist of clusters of strings - functionally independent sub-arrays. The deployment of the first demonstration cluster has been started in April 2013. In 2014 the deployment of the second stage of the demonstration cluster has been performed. We describe the configuration and design of the first GVD cluster and review the current status of cluster deployment in Lake Baikal.
\end{abstract}

\section{Introduction}

The next generation neutrino telescope Baikal-GVD in Lake Baikal will be a research infrastructure aimed primarily at studying astrophysical neutrino fluxes and particularly at mapping the high-energy neutrino sky in the Southern Hemisphere including the region of the galactic center. Baikal-GVD is a 3-dimensional lattice of optical modules (OMs) photomultiplier tubes enclosed in transparent pressure spheres. The OMs are arranged on vertical load-carrying cables to form strings. The telescope will consist of clusters of strings functionally independent sub-arrays, which are connected to shore by individual electrooptical cables. Each cluster comprises seven peripheral strings uniformly arranged at a $60 \mathrm{~m}$ distance around a central one. The OMs are spaced by $15 \mathrm{~m}$ along each string and facing downward. The OMs along a string are combined in sections - the functional detection unit of the telescope. The distances between the central strings of neighboring clusters are $300 \mathrm{~m}$.

${ }^{a}$ Corresponding author: djilkib@yandex.ru

(C) The Authors, published by EDP Sciences. This is an Open Access article distributed under the terms of the Creative Commons Attribution License 4.0 (http://creativecommons.org/licenses/by/4.0/). 
Two telescope configurations with 2304 OMs (GVD) and 10368 OMs (GVD*4) have been optimized. For GVD*4 the muon effective area rises from $0.3 \mathrm{~km}^{2}$ at $1 \mathrm{TeV}$ to about of $1.8 \mathrm{~km}^{2}$ asymptotically and the angular resolution (median angle between generated and reconstructed muon directions) is about 0.25 degree. The shower effective volumes of GVD*4 above $10 \mathrm{TeV}$ range from 0.4 to $2.4 \mathrm{~km}^{3}$. The accuracy of shower energy reconstruction is about $20-35 \%$ depending on shower energy, and the angular resolution is about 3.56.5 degrees (median value).

The site chosen for the experiment is in the southern basin of Lake Baikal. Here, the combination of hydrological, hydro-physical, and landscape factors is optimal for the deployment and the operation of the neutrino telescope. The water depth is about $1360 \mathrm{~m}$ at distances greater than three kilometers from the shore. The water transparency is characterized by an absorption length of about $20-25 \mathrm{~m}$ and a scattering length of $30-50 \mathrm{~m}$ [1]. The water luminescence is moderate at the detector site.

Prototypes of the GVD elements and systems have been tested in Lake Baikal in 20082010 as a part of the NT200+ detector [2-5]. The prototyping/early construction phase of the Baikal-GVD project which aims at construction and operation of the first demonstration cluster has been started in 2011 by deployment and operation of autonomous engineering arrays [6-9]. The next important step in the realization of the GVD project was made in 2013 by the deployment of an enlarged engineering array - the first stage of the demonstration cluster, which comprises $72 \mathrm{OMs}$ arranged on three $345 \mathrm{~m}$ long strings [10] and successfully operated from April 2013 to February 2014.

\section{Demonstration cluster}

The demonstration cluster will comprise a total of 192 optical modules arranged on eight $345 \mathrm{~m}$ long strings, as well as an acoustic positioning system [8] and an instrumentation string with equipment for the array calibration and the monitoring of environmental parameters. Seven side strings will be located at $60 \mathrm{~m}$ distances from a central one. Each string comprises 24 OMs spaced by $15 \mathrm{~m}$ at depths between $900 \mathrm{~m}$ and $1250 \mathrm{~m}$ below the surface. Each OM consists of a pressure-resistant glass sphere with $43.2 \mathrm{~cm}$ diameter which holds the OM electronics and the PMT which is surrounded by a high permittivity alloy cage for shielding it against the Earth magnetic field. A large photomultiplier tube Hamamatsu R7081-100 with a 10-inch hemispherical photocathode and quantum efficiency up to $35 \%$ has been selected as light sensor. Besides the PMT, an OM comprises a high voltage power supply unit (HV), a fast two-channel preamplifier, and a controller. For temporal and amplitude calibration of the measuring channel, two LEDs are installed in the optical module. The OM controller is intended for HV control and monitoring, for PMT noise measurements, and for time and amplitude calibration.

The optical modules are grouped into sections - the detection units of the array [11]. Each section includes $12 \mathrm{OMs}$ and the central module (CeM). PMTs signals from all OMs are transmitted through $90 \mathrm{~m}$ long coaxial cables to the CeM of the section, where they are digitized by custom-made ADC boards with $200 \mathrm{MHz}$ sampling rate. The waveform information from all measuring channels of the section is transferred to the Master board located in the CeM. The Master board provides the readout of the ADC data, the connection via local Ethernet to the cluster DAQ-center, the control of the section operation and the section trigger logic [11]. A request of the section trigger is transferred from the Master board to the cluster DAQ-center, where a global trigger for all sections is generated. The global trigger initiates data transmission from all sections to shore through a $6 \mathrm{~km}$ long electrooptical cable. 

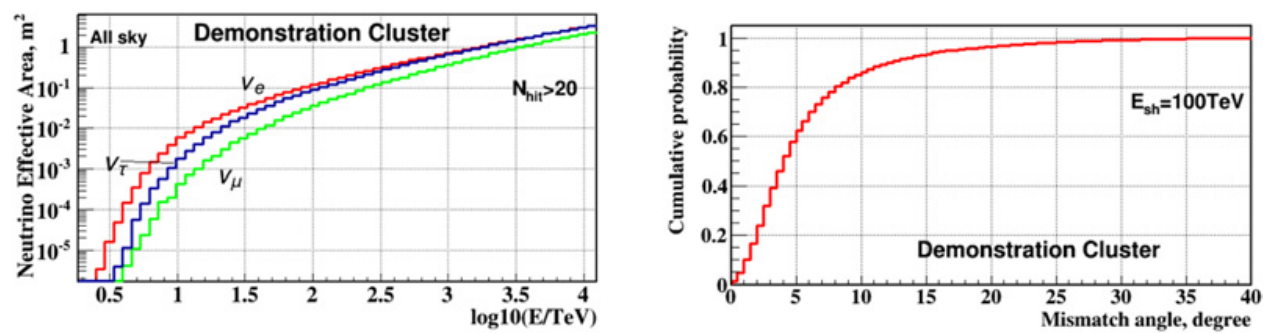

Figure 1. Left: neutrino effective areas for each flavor averaged over all arrival angles. The effective area includes effects from attenuation of neutrinos in Earth. Right: the fraction of reconstructed showers with mismatch angle less than a given value.
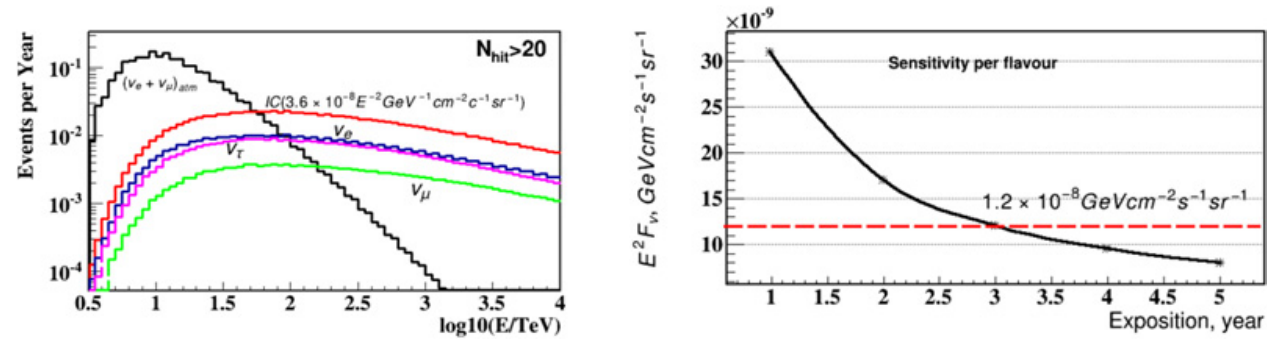

Figure 2. Left: expected distributions of events per year from astrophysical fluxes obtained by IceCube. Also shown is the expected distribution of background events from atmospheric neutrinos. Right: cluster sensitivity for one flavor neutrino flux with an $\mathrm{E}^{-2}$ spectrum as function of the observation years. The long-dashed line indicates the one flavor neutrino flux value obtained by IceCube.

The demonstration cluster has the potential to detect astrophysical neutrinos with a flux value measured recently by IceCube $[12,13]$. The search for high-energy neutrinos is based on the selection of cascade events generated by neutrino interactions in the sensitive volume of array. After applying an iterative procedure of vertex reconstruction followed by the rejection of hits contradicting the cascade hypothesis on each iteration stage [14], events with a final multiplicity of hit OMs $\mathrm{N}_{\text {hit }}<20$ are selected as high-energy neutrino events. With this event selection, the neutrino effective areas for each flavor assuming an equal flux of neutrinos and antineutrinos and averaged over all arrival angles have been derived (see, Fig. 1 left). These areas are about ten times smaller than the corresponding areas of IceCube [12]. The accuracy of shower direction reconstruction is about 4 degree (median value), which is substantially better than the 10-15 degree accuracy for IceCube [12]. The fraction of shower events $\left(\mathrm{E}_{\mathrm{sh}}=100 \mathrm{TeV}\right)$ with mismatch angles between generated and reconstructed muon directions less than a given value is shown in Fig. 1 (right). Energy distributions of the expected number of shower events per year for IceCube astrophysical fluxes for different flavors and all-flavor, as well as the distribution of expected background events from atmospheric neutrinos are shown in Fig. 2 (left).

The expected background events from atmospheric neutrinos are strongly suppressed for energies higher than $100 \mathrm{TeV}$. We expect about one event per year with $\mathrm{E}_{\mathrm{sh}}<100 \mathrm{TeV}$ from an equal-flavor astrophysical flux with a per-flavor normalization $\mathrm{E}^{2} \mathrm{~F}=1.2 \cdot 10^{-8} \mathrm{GeV}$ $\mathrm{cm}^{-2} \mathrm{~s}^{-1} \mathrm{sr}^{-1}$ [12] in the demonstration cluster, compared to about 10 events in IceCube. The sensitivity of one cluster for one flavor neutrino flux with an $\mathrm{E}^{-2}$ spectrum as function of the observation years is shown in Fig. 2 (right). Three year exposition allows sensitivity at a level of flux value measured by IceCube. 


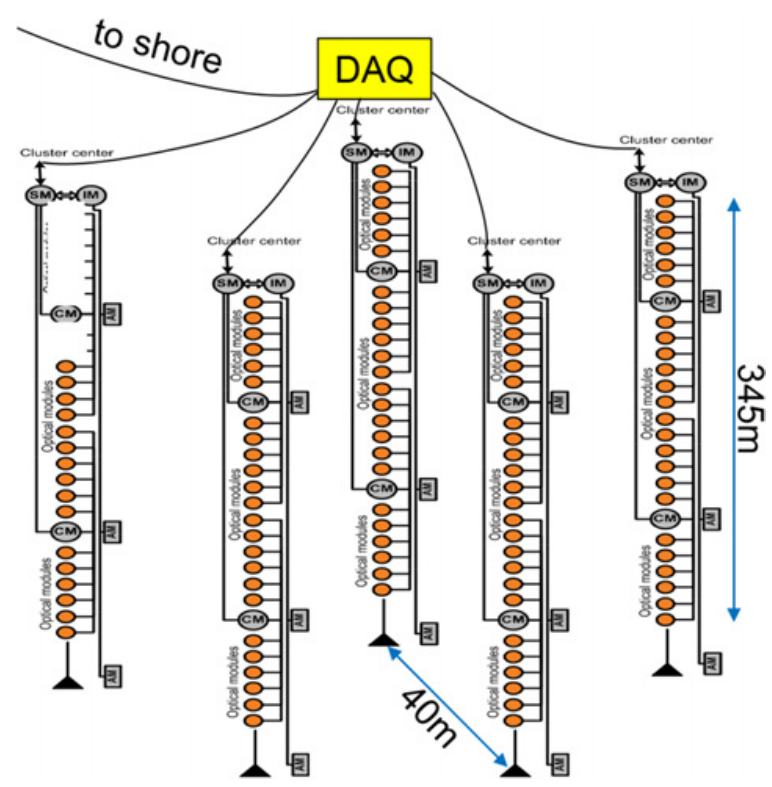

Figure 3. Schematic drawing of the second stage of the demonstration cluster.

\section{Current stage of the demonstration cluster}

The next important step in the realization of the GVD project was made in 2014 by deployment of the second stage of the demonstration cluster, which comprises $112 \mathrm{OMs}$ arranged on five strings, as well as an instrumentation string with array calibration and environment monitoring equipment. The schematic view of this array is shown in Fig. 3. The vertical spacing of OMs is $15 \mathrm{~m}$ and the horizontal distance between strings is about $40 \mathrm{~m}$. In addition to the OMs, each string comprises the communication module (CoM), and the two central modules $(\mathrm{CeM})$ of the two sections of the string. Also each string comprises four transceivers of the acoustic positioning system (AM). The modified cluster DAQ-center is located at the central string of the demonstration cluster and is connected to shore by the electro-optical cable.

The instrumentation string is located at $60-100 \mathrm{~m}$ away from the measuring strings with the OMs. It comprises the calibration laser source, eight optical modules, as well as 10 acoustic sensors of the positioning system. The calibration laser source is located at $1215 \mathrm{~m}$ depth and is used for time synchronization between the OMs on different strings. The high intensity of the laser source (up to $6 \cdot 10^{13}$ photons/pulse) allows the illumination of OMs at distances more than $200 \mathrm{~m}$ away from the source. The acoustic sensors are arranged along the instrumentation string starting from $50 \mathrm{~m}$ depth to the bottom of the string and perform monitoring of the string displacements at different depths caused by deep or/and surface water currents. Eight optical modules housing two R8055 and six XP1807 PMTs are arranged at depths from $600 \mathrm{~m}$ to $900 \mathrm{~m}$ on the instrumentation string and aim at monitoring of the light background at these depths.

The second stage of the demonstration cluster is successfully operating since April 2014 in several testing and data taking modes. In data taking mode a total of 2.96 . $10^{8}$ events has been recorded which corresponds to 166 days of live time from April 10 till December 30. Long-term control and monitoring of the array measuring system behaviour, as well as background conditions during the array operation in 2014 have been performed. 

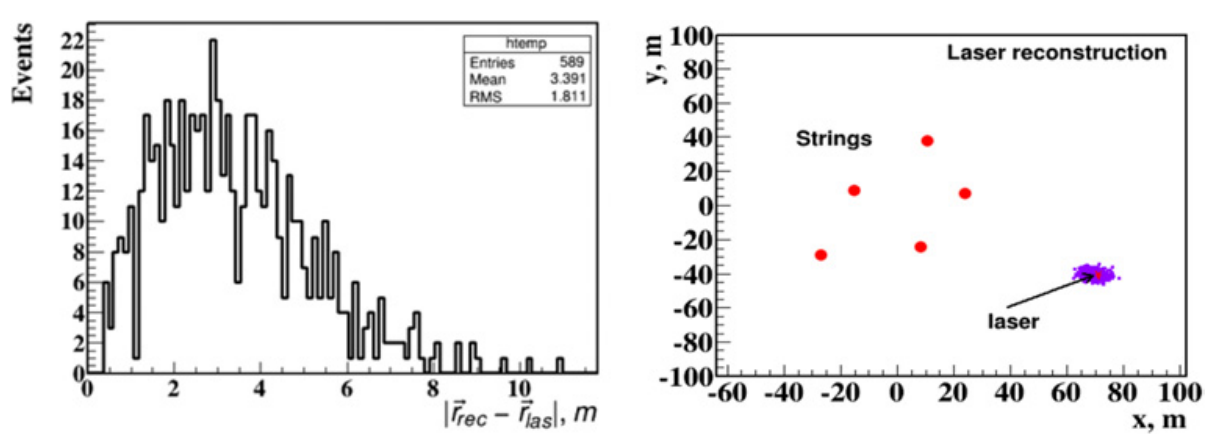

Figure 4. Left: deviation of reconstructed laser coordinates from those obtained by the acoustic positioning system. Right: top view of reconstructed laser positions. The star indicates the laser position obtained by the acoustic system.

The main contribution to the recorded counting rates comes from radiation produced by chemiluminescence in the deep water. During April - December 2014 the PMTs counting rates are about of $15-40 \mathrm{kHz}$, which are comparable with values obtained in 2012-2013.

One of the main goals of the array operation in testing modes is the estimation of the ability of in-situ calibration procedures. Calibration of the array recording system includes charge and timing calibrations of the measuring channels and time synchronization of OMs on different sections. All these calibration procedures are based on the usage of the OM's internal calibration LEDs and the external laser light source.

The performance and quality of the calibration procedures have been verified by the reconstruction of position and intensity of the calibration laser. An external calibration laser provides five series of $480 \mathrm{~nm}$ light pulses at five fixed intensity levels ranging from approximately $10^{12}$ to $6 \cdot 10^{13}$ photons/pulse, which roughly corresponds to shower energies from $10 \mathrm{PeV}$ to $600 \mathrm{PeV}$. This allows testing the array capability for high-energy cascade reconstruction. The position of the laser source was reconstructed using the arrival times of photons detected by the PMTs, taking into account the timing calibration of the PMTs by LEDs. Results of the reconstruction were compared with the laser coordinates obtained by the acoustic positioning system. Differences between reconstructed coordinates and those obtained by the acoustic system are shown in Fig. 4 (left). The reconstruction accuracy (median value) is about $3 \mathrm{~m}$. Shown in Fig. 4 (right) are strings and laser positions obtained by the acoustic system, as well as reconstructed laser positions. The obtained results demonstrate the expected quality of the array calibration procedures.

\section{Conclusion}

The ambition of the Baikal collaboration is the construction of a km3-scale neutrino telescope - the Gigaton Volume Detector in Lake Baikal. During the R\&D phase of the GVD project in 2008-2010, its basic elements - new optical modules, FADC readout units, underwater communications and trigger systems - have been developed, produced and tested in situ by long-term operating prototype strings. The prototyping phase of the GVD project has been started since April 2011 and aims at completion of the first demonstration cluster of GVD in spring 2015. The first stage of the GVD-cluster comprising three strings was deployed and successfully operated in 2013. In 2014 the second stage of the demonstration cluster with $112 \mathrm{OMs}$ at 5 strings has been deployed and is presently taking data. 
This work was supported by the Russian Found for Basic Research (grants 13-02-12221, 14-02-00175, 14-02-00972).

\section{References}

[1] A. Avrorin et al., Nucl. Instr. and Meth. A 693, 186 (2012)

[2] V. Aynutdinov et al., Nucl. Instr. and Meth. A 602, 227 (2009)

[3] A. Avrorin et al., Nucl. Instr. and Meth. A 626, S13 (2011)

[4] A. Avrorin et al., Nucl. Instr. and Meth. A 639, 30 (2011)

[5] A.V. Avrorin et al., Instruments and Experimental Techniques 54, 649 (2011)

[6] A. Avrorin et al., Nucl. Instr. and Meth. A 692, 46 (2012)

[7] A. Avrorin et al., Nucl. Instr. and Meth. A 725, 23 (2013)

[8] A.V. Avrorin et al., Instruments and Experimental Techniques 56, 449 (2013)

[9] A. Avrorin et al., J. of Phys. Conf. Series. 409, 012141 (2013)

[10] A. Avrorin et al., Nucl. Instr. and Meth. A 742, 82 (2014)

[11] A.D. Avrorin et al., Instruments and Experimental Techniques. 57, 262 (2014)

[12] M. G. Aartsen et al. [IceCube Collaboration], Science. 342, 1242856 (2013)

[13] M. G. Aartsen et al. [IceCube Collaboration], Phys. Rev. Lett. 113, 101101 (2014)

[14] A. Avrorin et al., Astronomy Lett. 35, 651 (2009) 